\title{
Perspektiven der Gesundheitspolitik im Neuen Europäischen Sozialmodell
}

\section{Marktintegration ohne Sozialpolitik}

Dass der soziale Integrationsprozess in Europa dem politischen, vor allem aber dem ökonomischen hinterher hinkt, ist mittlerweile fast ein Gemeinplatz. Die Vereinigung Europas ist im Kern ein ökonomisches Projekt, und noch dazu eines, das durch wirtschaftsliberale, angebotspolitische und monetaristische Leitbilder geprägt wurde. Die Verankerung und Ausgestaltung der individuellen und kollektiven sozialen Bürgerrechte war stets dem ökonomischen Integrationsprozess nachgeordnet (Jachtenfuchs/Kohler-Koch 1996, Leibfried/ Pierson 1998, Bieling/Steinhilber 2000a). Der Europäische Binnenmarkt sowie die Europäische Wirtschafts- und Währungsunion sind Realität, auf einen europäischen Wohlfahrtsstaat wagen gegenwärtig nicht einmal chronische Optimisten zu hoffen.

Diese integrationspolitische Asymmetrie, die in der absoluten Nachrangigkeit der sozialen Dimension im europäischen Integrationsprozesses zum Ausdruck kommt, hat unterschiedliche Ursachen. Zum einen ist sie darauf zurückzuführen, dass die großen „hegemonialen Projekte“ (Bieling/Steinhilber 2000b), deren Realisierung die europäische Integration voran getrieben und geprägt hat, allesamt ökonomischer Natur waren. Sie dienten der Integration von Märkten in Europa und ihrer wirtschafts- und finanzpolitischen Flankierung bzw. der Stärkung des "Wirtschaftsstandortes Europa“. Wohlfahrtsstaatliche Handlungsspielräume wurde eher eingeschränkt oder spielten - im günstigsten Fall keine Rolle. Dies gilt erstens für das 1979 etablierte Europäische Währungssystem (EWS). Nach dem Zusammenbruch des Bretton-Woods-Systems wurde es als finanzpolitische Antwort auf die negativen Folgen stark schwankender Wechselkurse sowie die damalige Weltwirtschaftskrise errichtet. Es sollte vor allem den innereuropäischen Handel auf eine stabilere Grundlage stellen. Es gilt zweitens gleichermaßen für das Europäische Binnenmarktprojekt, das als Reaktion auf die zunehmende Schwäche der europäischen Ökonomien gegenüber den Zentren der kapitalistischen Triade (in Nordamerika und Ostasien) zu werten ist. Zwar kam es im Rahmen des Binnenmarktprojektes auch $\mathrm{zu}$ einigen sozialen Mindestregelungen, aber im Vordergrund stand die Ab- 
schaffung nichttarifärer Handelshemmnisse in den Waren-, Dienstleistungs-, Kapital- und Arbeitsmärkten sowie die wechselseitige Anerkennung nationaler Standards. Drittens spielten auch bei der Errichtung der Wirtschafts- und Währungsunion und des Euro-Finanzregimes nicht sozialpolitische, sondern wettbewerbs- und finanzpolitische Motive die entscheidende Rolle. Durch die einseitige Orientierung der europäischen Zentralbank auf die Geldwertstabilität und die restriktiven Defizit- und Schuldenkriterien des Europäischen Wachstums- und Stabilitätspaktes wurde sogar, nicht zuletzt auf Druck der deutschen Bundesregierung und der deutschen Bundesbank, ein Finanzregime errichtet, in dem den nationalen Wohlfahrtsstaaten Knebel angelegt und die Interessen der institutionellen Finanzmarktakteure kursbestimmend wurden. Und viertens schließlich dürfte die Subordination der Sozialpolitik auch für das nächste politische Großprojekt, die angestrebte Integration der europäischen Finanzmärlte gelten (Bieling/Steinhilber 2002). Diesem Projekt liegt letztlich das Leitbild eines finanz(markt)getriebenen Entwicklungsmodells zugrunde, das allmählich als hegemoniales Regime globale Konturen gewinnt (Aglietta 2000, Boyer 2000). Es orientiert sich am neoamerikanischen Kapitalismusmodell (Albert 2001), vor allem an den Sektoren der „New Economy“. Verbunden ist damit die Hoffnung auf mehr Wettbewerbsfähigkeit für Euro$\mathrm{pa}$, ein verbessertes Investitionsklima für die Unternehmen und mehr Beschäftigung. Zur Zeit wird dieses Projekt durch entsprechende Rechtsetzungsakte, Richtlinien und politische Initiativen auf den Weg gebracht.

Diese „Marktintegration ohne oder gar gegen Sozialpolitik“" wurde und wird durch die Regierungen der Mitgliedsstaaten vorangetrieben. Sie sind und bleiben auf absehbare Zeit die Schlüsselakteure des europäischen Integrationsprozesses. Dabei prägen zweifelsohne die strategischen Interessen der europäisch agierenden Großkonzerne sowie der global ausgerichteten Finanzmarktakteure an einer ökonomischen Sphäre, in der die Realisierung von Profit- und Zinsoptionen ohne sozialregulative Fesseln möglich ist, die Ausrichtung des Integrationsprozesses. Doch auch die Nationalstaaten zeigten lange Zeit wenig Fähigkeit oder eigene Interessen daran, die Sozialpolitik in Europa bzw. die Entwicklung der nationalen Wohlfahrtsstaaten zu einem europäischen Thema zu machen (Streeck 1998, Scharpf 1999). Das galt für „rechte" wie für „linke" Regierungen, freilich mit eigenen Begründungen. Rechte Regierungen wollten sozialpolitische Kompetenzen auf EU-Ebene verhindern, um nicht von der europäischen Seite her soziale Mindestvorgaben für ihre nationalen Deregulierungs- und Privatisierungsprojekte befürchten zu müssen. ${ }^{1}$ Aber auch eher linke Regierungen wollten die Sozialpolitik als nationalstaatliches Thema erhalten. Vor allem, weil sie aus dem wirtschaftsliberalen Europa für die nationalen

1 Margaret Thatcher wurde schon bei dem Wort „soziale Grundrechte“ von dem Alptraum geplagt, über die Brücke Europa würde der Sozialismus in Großbritannien Einzug halten. 
Wohlfahrtsstaaten nichts Gutes erwarteten. Sie befürchteten Deregulierungsund Privatisierungsvorgaben, die ihnen das Geschäft der nationalen Wohlfahrtsstaatsreformen weiter erschweren würde. Wie wir wissen, waren diese Befürchtungen durchaus realistisch.

Nun soll keineswegs übersehen oder gering geschätzt werden, dass es in den 90er Jahren durchaus Fortschritte im Bereich der europäischen Sozialpolitik gab (Falkner 1998, Schulte 2001, Kruse/Geisen 2001). Durch den Vertrag von Amsterdam wurde das ehemalige "Sozialpolitische Protokoll' ${ }^{\text {is }}$ in den Vertragstext übernommen, der EG-Vertrag erhielt ein „Beschäftigungs-Kapitel“, im Bereich "Gesundheit und Sicherheit am Arbeitsplatz" wurden einige fortschrittliche Richtlinien erlassen, und bei der Bekämpfung von „Armut und sozialer Ausgrenzung" entwickelte die EU Aktivitäten. Doch nach wie vor hinken die sozial- und beschäftigungspolitischen Maßnahmen der ökonomischen Integration hoffnungslos hinterher und sind die institutionellen Hürden in wichtigen Feldern der Sozialpolitik hoch. So setzen Ratsentscheidungen im Bereich der "sozialen Sicherheit und des sozialen Schutzes" nach wie vor Einstimmigkeit voraus und enden somit fast zwangsläufig in jenen Politikverflechtungsfallen, die der Sozialpolitik im europäischen Mehrebensystem seit jeher das Leben schwer machen (Scharpf 1985).

\section{Die Lissabonner Wettbewerbsstrategie und die "offene Methode der Koordinierung"}

Es steht also zu befürchten, dass sich auch der bevorstehende Integrationsschub in Europa ohne hinreichende sozialpolitische Interventionen vollziehen wird. Wenn sich Europa auf den Weg zum „wettbewerbsstärksten, wissensbasierten Wirtschaftsraum" der Welt macht, wie es der Europäische Rat auf seiner Sondersitzung im März 2000 in Lissabon (Europäischer Rat 2000) beschlossen hat, wird dies erneut und wahrscheinlich noch stärker als bisher mit sozialen Verwerfungen einhergehen. Denn zweifelsohne steht die Systemkonkurrenz zwischen dem europäischen und dem neoamerikanischen Kapitalismusmodell und die Stärkung der Attraktivität des Wirtschafts- und vor allem des Kapitalraumes Europa im Vordergrund.

Alles Gesagte bedeutet jedoch nicht, dass im nächsten Integrationsschub nach den Leitbildern der „Lissabonner Wettbewerbsstrategie" Sozialpolitik keine Rolle spielt oder sozialpolitische Politikfelder unberührt bleiben. Vor allem die Auswirkungen auf die nationalen Wohlfahrtsstaaten werden massiv sein. Denn die neue wettbewerbspolitische Formierung Europas versucht gerade die Politikfelder, die in Europa oder den Mitgliedsstaaten für die wohlfahrtsstaatliche Entwicklung wichtig sind, in ihren Dienst zu stellen und der wettbewerbspolitischen Zielsetzung unterzuordnen. Das gilt für die Finanzpolitik, die fast ausschließlich auf einen strikten Kurs der Konsolidierung der öffentli- 
chen Haushalte gerichtet ist, gleichgültig, ob dies die konjunkturellen und Wachstumsprobleme in den Ländern Europas verschärft, die ohnehin unzureichende öffentliche Investitionsquote weiter absenkt und damit dem weiteren Verfall der öffentlichen Infrastruktur Vorschub leistet. Es gilt auch für die Geldpolitik. Sie wird durch die vertraglich fixierten Kompetenzen und Aufgaben der Europäischen Zentralbank als Instrument einer aktiven Konjunktur-, Wachstums- und Beschäftigungspolitik entwertet und ausschließlich auf die Geldwertstabilität ausgerichtet. Damit dient sie letztendlich vor allem den Einkommens- und Sicherheitsinteressen der großen Geldvermögensbesitzer in Europa. Es gilt ebenfalls für die Aktivitäten im Bereich der Arbeitnehmerschutzrechte. Sie sollen umfassend dereguliert und flexibilisiert werden, um den betrieblichen Arbeitskräfteeinsatz ohne Reibungsverluste an die neuen Produktionsmodelle anpassen zu können. Vor allem der Kündigungsschutz wird mit großer Vehemenz infrage gestellt. Es gilt ebenfalls für die europäische Arbeitsmarktpolitik, die durch Deregulierung und Flexibilisierung von den Arbeitsmärkten her den Umbau des alten Produktionsmodells flankieren soll; hier stehen die Ausdehnung ungeschützter Formen der Lohnarbeit, vor allem von Zeit- und Leiharbeit sowie die angeblich unzureichende Beschäftigungsfähigkeit im Vordergrund. Und schließlich gilt es für die Reform der Systeme des Sozialschutzes, die ebenfalls zur Konsolidierung der öffentlichen Haushalte beitragen und die Schritt für Schritt, aber sehr systematisch für die Anlageinteressen der Finanzmarktakteure geöffnet werden sollen.

\subsection{Die Lissabonner Wettbewerbsstrategie als strategisches Programm}

Im Leitbild der „Lissabonner Wettbewerbstrategie" erfährt die Sozialpolitik also durchaus eine strategische Aufwertung, freilich nicht als marktkorrigierende, sonder als marktöffnende und -fördernde Politik (zu dieser Unterscheidung etwa Scharpf 1999). Angesichts der Herausforderungen der Globalisierung und im Banne der Dynamik der US-amerikanischen Ökonomie müsse sich die Union auf ein „klares strategisches Ziel festlegen und sich auf ein ambitioniertes Programm für den Aufbau von Wissensinfrastrukturen, die Förderung von Innovation und Wirtschaftsreform und die Modernisierung der Sozialschutz- und der Bildungssysteme einigen." (Europäischer Rat 2000: 1). Dieses neue strategische Ziel besteht nun darin, „die Union zum wettbewerbsfähigsten und dynamischsten wissensbasierten Wirtschaftsraum der Welt zu machen - einem Wirtschaftsraum, der fähig ist, ein dauerhaftes Wachstum mit mehr und besseren Arbeitsplätzen und einem größeren sozialen Zusammenhalt zu erzielen" (Europäischer Rat 2000: 2). Ergänzt wurde diese Zielsetzung durch die Rehabilitierung des Vollbeschäftigungsziels und die Vereinbarung eines "makroökonomischen Policy-Mix", durch den das Ziel eines durch- 
schnittlichen Wirtschaftswachstums von etwa 3\% bis zum Jahre 2010 realisiert werden sollte. Darüber hinaus wurde die im europäischen Stabilitäts- und Wirtschaftspakt formulierte Zielsetzung einer strikten und nachhaltigen Konsolidierung der öffentlichen Finanzen bekräftigt und zugleich die Notwendigkeit einer umfassenden „Modernisierung des sozialen Schutzes" betont. Dabei soll die Anpassung der Sozialschutzsysterne an die Anforderungen der wissensbasierten Wirtschaft den strategischen Transformationsprozess des ökonomischen Entwicklungsmodells aktiv unterstützen. Der Rat und die Kommission wurden aufgefordert, nach dem üblichen Verfahren bis zum Frühjahr 2001 einen Bericht vorzulegen, in dem anhand vergleichbarer Daten und Indikatoren überprüft werden sollte, ob in den Mitgliedsstaaten angemessene konkrete Schritte unternommen werden, „um (...) die langfristige Nachhaltigkeit der öffentlichen Finanzen sicherzustellen, indem (...) alle diesbezüglichen Aspelkte, einschließlich der Auswirkungen der Alterung der Bevölkerung, geprüft werden" (Europäischer Rat 2000: 8). Dabei wurden sowohl die Förderung von Sach- und Humankapital als auch die Gewährleistung einer guten Gesundheitsfürsorge als strategische Ziele benannt.

Nun muss bereits mit geringem zeitlichem Abstand konstatiert werden, dass sowohl die ökonomischen als auch die beschäftigungspolitischen Zielsetzungen der Lissabonner Strategie offensichtlich zu ambitioniert ausfielen. Weder befinden sich die nationalen Ökonomien in der EU auf einem mittelfristigen Wachstumspfad von 3\%, noch ist das Ziel der Vollibeschäftigung auf absehbare Zeit erreichbar. Und obwohl die US-amerikanische wie die europäische "New Economy" zu Beginn dieses Jahrhunderts in tiefe Rezessionen fielen, hat sich die ökonomische Gesamtpotenz der USA gegenüber dem Euro-Raum wohl eher vergrößert als verringert. ${ }^{2}$ Somit steht zumindest aus der anvisierten Zeitperspektive das gesamte politische Projekt des Lissabonner Gipfels infrage. Gleichwohl sollte Gleiches nicht vorschnell für die Strategie behauptet werden, im Kernbereich der EU über eine koordinierte, politische Strategie den Übergang vom fordistischen Industrie- zum flexiblen Finanzkapitalismus zu befördern (Bieling li.a. 2001). Es spricht vielmehr einiges dafür, dass die ökonomische Stagnationsphase, in die einige der Kernstaaten des Euro-Raumes geraten sind, die politische Entschlossenheit zur Durchsetzung der strategischen Ziele des Lissabonner Projektes eher verstärkt als entmutigt hat. ${ }^{3}$

2 Während das BIP in den USA zwischen 2000 und 2002 um 6,5\% stieg, wuchs es im EuroRaum lediglich um 5,7\% (Sachverständigenrat 2002, Tab. 4 und 8).

3 Auch die umstrittene „Agenda 2010" von Bundeskanzler Gerhard Schröder ist als Versuch der nationalstaatlichen Umsetzung der Lissabonner Strategie zu werten. So heißt es in dem Leitantrag „Mut zur Veränderung“, der auf dem SPD-Sonderparteitag vom 1. Juni 2003 angenommen wurde: „Die Agenda 2010 ist ein sozialdemokratisches Programm für Wachstum und Beschäftigung. Sie steht im Einklang mit dem Beschluss der europäischen Staats- und Regierungschefs von Lissabon, die Europäische Union innerhalb von 10 Jahren zur dynamischsten und wettbewerbsfähigsten Wachstumsregion weltweit auszubauen und die Voraussetzungen für Vollbeschäftigung in der Europäischen Union zu schaffen." 


\subsection{Die "offene Methode der Koordinierung" als neuer Regulierungstyp}

Zum Zwecke einer effizienten Umsetzung der strategischen Beschlüsse von Lissabon einigte sich der Europäische Rat bereits auf dem Lissabonner Gipfel auf die Anwendung eines neuen Koordinierungsverfahrens. Dies geschah mit dem expliziten Ziel, seine Leitungs- und Koordinierungsfunktion bei der Umsetzung des strategischen Generalziels einer wettbewerbsfähigen Wissensökonomie sowie der strategischen Unterziele zu stärken. Bei dem neuen PolitikModus handelt es sich um die so genannte „offene Methode der Koordinierung" (OMK), die dem Europäischen Rat im Prozess der wettbewerbspolitischen Restrukturierung des Euro-Raumes eine stärkere Koordinierungs- und Leitungsfunktion zuweist (Europäischer Rat 2000: 12/13). Bei der OMK handelt es sich um ein eigenständiges, jenseits des Erlasses von Richtlinien und Verordnungen angesiedeltes Abstimmungs- und Umsetzungsverfahren des Europäischen Rates, in dem über die Analyse quantitativer und qualitativer Indikatoren „bewährte Praxisbeispiele" („best practice") identifiziert und in Benchmarks transformiert werden. Diese sollen in strategische Leitlinien eingehen, zu deren Umsetzung konkrete Zeitpläne sowie regelmäßige Überwachungs-, Bewrertungs- und Prüfungsverfahren (Monitoring-Verfahren) vereinbart werden. Bei der Einrichtung eines entsprechenden Überwachungssystems soll die Evaluierung des Umsetzungsfortschritts mit Hilfe von Ranking-Listen und einer Peer-Group-Review geschehen. ${ }^{4}$ Die OMK soll, wie auf diversen Nachfolgegipfeln festgelegt wurde, Anwendung auf nahezu allen wettbewerbspolitisch relevanten Politikfeldern finden (Hodson/Maher 2001, Ohndorf 2002, Hill 2002, Schulz-Weidner 2002). Zu diesen werden auch die Alterssicherungspolitik (Eckardt 2002, Verband Deutscher Rentenversicherungsträger u.a. 2002), die Maßnahmen gegen „Armut und soziale Ausgrenzung" (Schulte 2002) und eben auch die Gesundheitspolitik (Busse 2002, Pitschas 2002) gezählt.

Zweifelsohne liegt dem Konzept der OMK der Versuch zugrunde, einen interessenpolitischen Spagat zwischen den Schlüsselakteuren der unterschiedlichen Ebenen zu meistern. Konzeptionell ,geht es bei der offenen Koordinie-

4 Integrationstheoretisch betrachtet scheint die OMK dem Idealtypus eines „autonomieschonenden und gemeinschaftsverträglichen" (F.W. Scharpf) Modus politischer Interaktion sehr nahe zu kommen. Diesem wird der Vorzug der Überwindung von Blockaden und Verflechtungsfallen im politischen Mehrebenen-System zugeschrieben. Ein solcher Modus stellt ein Modell nationalen und gemeinschaftlichen Regierens dar, das in wechselseitiger Rücksichtnahme auf jeweils autonome Kompetenzen, Interessenlagen und Problemlösungskapazitäten vor allem auf eine möglichst effektive Entscheidungsfindung ausgerichtet ist. „Die Gemeinschaft dürfte danach ihre jeweiligen Ziele nur mit Mitteln verfolgen, welche die kulturelle und institutionelle Integrität und die Autonomic der internen Willensbildung in den Mitgliedsstaaten möglichst wenig beeinträchtigt. Umgekehrt dürften aber auch die Mitgliedsstaaten und subnationalen Gebietskörperschaften die eigenen Ziele nur mit gemeinschaftsverträglichen Mitteln verfolgen." (Scharpf 1994: 139). 
rung nicht um Eingriffe in die Rechtsetzung und Verwaltungshoheit der Mitgliedsstaaten, sondern es geht darum, dass sich die Mitgliedsstaaten in ihrer Politik freiwillig auf politische Ziele einigen“; wobei „Rücksicht genommen werden (muss) auf die politischen Möglichkeiten des einzelnen Mitgliedsstaa-

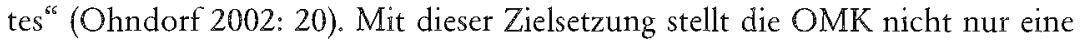
einfache Ergänzung der bisher praktizierten Modalitäten europäischer Politik dar. Sie scheint die Interessen der EU an weiterreichenden Kompetenzen in der Sozialpolitik mit den Interessen der Mitgliedsstaaten, ihre Kompetenzen nicht aufzugeben, zu verbinden. Dieser Dualismus aus strategischer Akzeptanz der nationalstaatlichen Souveränitätsansprüche und einem Verfahren zur weitgehenden inhaltlichen Abstimmung der Sozialpolitiken macht sie zu einer regulativen „Maßanfertigung" (Raul Briet), die passgenau auf die interessenpolitische und institutionelle Konfiguration in der EU zugeschnitten ist.

\section{Europäisierung der Gesundheitspolitik?}

\subsection{Gesundheitspolitik im bisherigen Integrationsprozess}

Es spricht einiges dafür, dass auch die Sozialpolitik in Europa über dieses neue Koordinierungsverfahren neuen Schwung erhalten wird; zugleich ist wahrscheinlich, dass der wettbewerbs- und finanzpolitische Kontext sie auf einen Entwicklungspfad lenken wird, der weniger auf die Europäisierung sozialer Standards und Leistungen als vielmehr auf einen Rückbau und eine Privatisierung der nationalen Sozialsysteme hinauslaufen dürfte. Analoges dürfte für die Gesundheitspolitik gelten.

Bis weit in die jüngste Vergangenheit hinein konnte von Gesundheitspolitik als einem europäischen Politikfeld nicht die Rede sein. Die Regierungen der Mitgliedsstaaten nahmen für sich in Anspruch, die Gesundheitspolitik in den nationalen Politikarenen und damit in ihrem Autonomiebereich zu halten. Dies wurde nicht zuletzt durch die rechtlichen und institutionellen Regeln im europäischen Primärrecht abgesichert, das die Gesundheitspolitik weitgehend als Angelegenheit der Mitgliedsstaaten definiert und der Union entweder keine Entscheidungskompetenzen zubilligt oder europäische Akteure (vor allem den Europäischen Gerichtshof) weitgehend auf Maßnahmen einer negativen Integration im Sinne einer nationalstaatlich deregulierenden und marktöffnenden Politik festlegt. Dass sich diese Konstellation recht gut mit dem relativ geringen Verflechtungsgrad der nationalen Gesundheitsmärkte, der institutionellen Vielfalt der Gesundheitssysteme und den unterschiedlichen normativen Präferenzen in den europäischen Sozialpolitiken vertrug, stabilisierte sie zusätzlich (ausführlicher Urban 2003).

Doch das im europäischen Primärrecht gezeichnete Bild einer uneingeschränkten Autonomie der Mitgliedsstaaten in Fragen der Gesundheitspolitik verzerrt bereits heute die Wirklichkeit. Auch in diesem Politikfeld hat der Prozess der „funktionale(n) Verflechtung von europäischer Integration und 
nationaler Sozialpolitik“ (Leibfried 2000: 81) längst eingesetzt, und auch hier waren es vor allem Entwicklungen, die als funktionale Reflexe auf die voranschreitende Realisierung der marktbezogenen Grundfreiheiten der Europäischen Verträge (,freier Verkehr von Waren, Personen, Dienstleistungen und Kapital ${ }^{6)}$ gewertet werden können. Man muss die gesundheitspolitische Autonomie der Mitgliedsstaaten nicht gleich zur "Fiktion “ (Busse 2002: 7) erklären, um zu konstatieren, dass sich jenseits der relativ klaren primärrechtlichen Kompetenzverteilung zwischen Gemeinschaft und Mitgliedsstaaten seit längerem weitaus intensivere Interdependenzen herausgebildet haben, als auf den ersten Blick sichtbar sein mögen. Von ihnen gingen bereits in der Vergangenheit verstärkte Integrationseffekte aus; und zugleich scheinen sie in der Gegenwart als Anknüpfungspunkte für das neu erwachte Gemeinschaftsinteresse an einer eigenen Regulierungskompetenz im Politikfeld Gesundheit zu dienen. Vor allem die ausgreifende Dynamik der europäischen Marktintegration könnte in naher Zukunft prägend auch in die Gesundheitspolitik ausstrahlen. Als wichtigstes Integrationsvehikel könnte sich die drohende Kollision zwischen dem europäischen Markt- und dem deutschen Sozialrecht erweisen, die Schritte in Richtung einer negativen Integration erzwingen könnte (vgl. Bieback 1999, 2001, Ebsen 2000, Hirsch 2000, Dudey u.a. 2002).

In zwei Urteilen bestätigte der Europäische Gerichtshof (EuGH) zwar, dass das Gemeinschaftsrecht die Zuständigkeit der Mitgliedsstaaten zur Ausgestaltung ihrer Sozialschutzsysteme unberührt lässt; gleichwohl verpflichtet er diese auf die Beachtung des Gemeinschaftsrechts bei der Ausübung der nationalen Regelungskompetenz. Der EuGH stellt fest, dass der freie Waren- und Dienstleistungsverkehr zwischen den Mitgliedsstaaten beeinträchtigt wird, wenn für Versicherte der Zugang zu Heilmitteln und ärztlichen Leistungen im EGAusland erschwert wird, was durch den Genehmigungsvorbehalt im deutschen Gesundheitsrecht der Fall ist. Zwar hat der EuGH einen solchen Vorbehalt bei einer sonst drohenden „erhebliche(n) Gefährdung des finanziellen Gleichgewichts der sozialen Sicherheit" faktisch für zulässig erklärt. Doch die hervorgehobene, hoch bemessene Bewertung der Freizügigkeitsvorgaben steht in einem deutlichen Spannungsverhältnis zu konstitutiven Regulierungselementen im deutschen System. Da die Kostenerstattung für im EG-Ausland in Anspruch genommene Leistungen grundsätzlich mit dem Sachleistungsprinzip der deutschen GKV und die Inanspruchnahme von Leistungen außerhalb des Geltungsbereichs der Versorgungsverträge zwischen den Verbänden der Krankenkassen und der Ärzte schnell mit der vertraglichen Leistungsregulierung in Konflikt gerät, wohnt der Entscheidungsbegründung eine Logik inne, aus der sich erhebliche Bedrohungen für das Sachleistungsprinzip sowie das System der Kollektivverträge im deutschen System ergeben können. ${ }^{5}$

5 Das Sachleistungsprinzip und das System der Kollektivverträge stellen zwei zentrale Eckpfeiler der sozialen Krankenversicherung in Deutschland dar. Im Gegensatz zur privaten Kran- 
Die zweite Gefährdung ergibt sich aus dem europäischen Wettbewerbsrecht, das insbesondere der sozialen Selbstverwaltung im deutschen System zu schaffen machen könnte (vgl. Hänlein/Kruse 2000, Ebsen 2000, Bieback 2001). Dabei geraten weniger einzelne Leistungen als das ganze gesundheitspolitische Regulierungssystem unter Druck. Das europäische Wettbewerbsrecht, dem unmittelbare Geltung in den Mitgliedsstaaten zukommt, enthält vor allem das Verbot wettbewerbsbeschränkender Vereinbarungen und Verhaltensweisen (Art. 81-89 des Vertrags über die Europäische Gemeinschaft [EGV]). Als solche sind insbesondere Maßnahmen zu sehen, die Beeinträchtigungen des Handels zwischen den Mitgliedsstaaten und eine Verhinderung, Einschränkung oder Verfälschung des Wettbewerbs innerhalb des Gemeinsamen Marktes bezwecken oder bewirken (können). Im Einzelnen geht es um die Verbote des Zusammenschlusses zu marktbeherrschenden Unternehmen, von Kartellabsprachen, von Diskriminierungen durch marktbeherrschende Unternehmen und um das Verbot der Beeinträchtigung des Wettbewerbs in der Gemeinschaft durch öffentliche Beihilfen. Für das Gesundheitssystem und vor allem mit Blick auf die Krankenkassen und Leistungserbringer bzw. ihre Verbände sind vor allem die Verbote der Kartellabsprachen und der Diskriminierung durch marktbeherrschende Unternehmen relevant. Ob dass EG-Wettbewerbsrecht mit allen seinen Implikationen jedoch Anwendung auf das deutsche Krankenversicherungssystem findet, hängt vor allem von zwei Fragen ab. Zum einen davon, ob bzw. wann und in welchen Konstellationen die Krankenkassen, ihre Verbände oder andere Selbstverwaltungsträger der GKV als Unternehmen oder Unternehmensverbände im Sinne des Gemeinschaftsrechts zu werten sind, und ob zweitens - für den Fall einer Bejahung dieser Frage - die eigentlich als wettbewerbsunverträglich verbotenen Verhaltensweisen der Selbstverwaltungskörperschaften der GKV nicht doch gerechtfertigt sind, weil ihnen die Erfüllung besonderer Aufgaben übertragen wurde. ${ }^{6}$

kenversicherung, in der das so genannte Kostenerstattungsprinzip gilt, werden in der gesetzlichen Krankenversicherung (GKV) nahezu 90\% der Leistungen für den weit überwiegenden Teil der Versicherten nach dem Sachleistungsprinzip, d.h. in Form von Sach- und Dienstleistungen erbracht, ohne dass der Patient zur Erstattung der entstehenden Kosten verpflichtet wäre. Zwischen Patienten und Ärzten (oder anderen Leistungserbringern) bestehen keine direkten ökonomisch-finanziellen Beziehungen. Die Kostenerstattung wird über Kollektivverträge zwischen (den Verbänden der) Krankenkassen und (denen der) Leistungserbringer geregelt. Der Versicherte erwirbt durch seine einkommensabhängigen Beitragsleistungen einen Versicherungsschutz, der ihm eine ausschließlich am Bedarfsprinzip orientierte Versorgung zusichert. Diese Struktur entlastet zum einen die Versicherten von der Verpflichtung, für die mitunter sehr hohen Behandlungskosten in Vorlage zu treten; zum anderen entkoppelt es die einkommensbezogene Beitragsleistung von der bedarfsorientierten Leistungsgewährung und schafft damit die Basis für den GKV-internen Solidarausgleich (Rosenbrock/Gerlinger 2003).

6 Gegenüber den Schlïsselakteuren des deutschen Gesundheitssystems gewinnt diese Problematik ihre besondere Brisanz durch deren Charakter als intermediäre Organisationen in einer spezifischen Mittellage zwischen Staat und Markt. Das deutsche System der staatlich regulierten sozialen Selbstverwaltung ist daher weitaus schwieriger in dieser rechtlichen Konstellation 
Insgesamt zeichnet sich somit eine deutliche Ausstrahlung der Europäischen Integration in das Regulierungssystem des deutschen Gesundheitswesens ab. Zum einen dürfte die Kollision zwischen dem europäischen Markt- und dem deutschen Gesundheitsrecht den Druck auf die Akteure, insbesondere den Gesetzgeber erhöhen, bei zukünftigen Reformen die Konformität mit dem Gemeinschaftsrecht der EG in den politischen Zielkatalog mit aufzunehmen. Dabei weisen die Vorgaben der europäischen Grundsätze zum freien Warenund Dienstleistungsverkehr sowie die des Wettbewerbsrechts zweifelsohne in Richtung Liberalisierung, Deregulierung und Privatisierung des deutschen Gesundheitssystems, denen gerade jene Strukturelemente zum Opfer fallen könnten, die für den Solidarcharakter der GKV stehen. Dies gilt vor allem für die Regulierung der Produktion und Verteilung der Gesundheitsgüter über das Sachleistungsprinzip und das System kollektiver Verträge zwischen Kassenverbänden und Leistungsanbietern. Zweitens zeichnet sich mit dem EuGH ein neuer „externer" Akteur in der deutschen Gesundheitspolitik ab, der trotz der primären Zuständigkeit der Nationalstaaten für die Gesundheitsversorgung zwar subsidiär, aber von außen und mit weitreichenden Folgen in das gesundheitspolitische Regulierungssystem interveniert. Auf jeden Fall schränkt der durch den EuGH markierte Korridor, innerhalb dessen sich die nationalen Wohlfahrtsstaaten zu bewegen haben, ihre reformpolitische Autonomie stärker ein, als dies auf den ersten Blick erscheinen mag. Das gilt insbesondere für das deutsche System der sozialen Selbstverwaltung. Will der Staat dieses System nicht grundsätzlich gefährden, hat er sich mit seinen Reformoptionen im Kontinuum des EG-Rechts zu bewregen.

\subsection{Die OMK in der Gesundheitspolitik}

Die OMK könnte die Ausstrahlung des europäischen Integrationsprozesses auf die deutsche Gesundheitspolitik erheblich verstärken. In der Gesundheitspolitik gewinnt sie besondere Bedeutung durch eine Mitteilung der Kommission zum Thema „Die Zukunft des Gesundheitswesens und der Altenpflege: Zugänglichkeit, Qualität und langfristige Finanzierbarkeit sichern" (Europäische Kommission 2001), die dem Europäischen Rat von Barcelona (März 2002) vorgelegt worden war. Diese Mitteilung dürfte sich als eine Art Gründungsdokument einer europäischen Gesundheitspolitik erweisen. In ihr versucht die Kommission an das sozialpolitische Essential der Lissabonner Strategie anzuknüpfen, die Systeme des Sozialschutzes den Erfordernissen des neuen, wissensbasierten Entwicklungsmodells anzupassen und gleichzeitig eine hochwertige medizinische Versorgung der Bevölkerung in Europa sicherzustel-

zu verorten als steuerfinanzierte, nationale Gesundheitssysteme oder weitgehend privatisierte Marktmodelle, in denen die Unternehmenseigenschaft einzelner Akteure offensichtlicher festgestellt oder verworfen werden kann. 
len. Auf dieser Grundlage hatte der Europäische Rat von Göteborg (Juni 2001) den Auftrag formuliert,

„gemäß dex offenen Koordinierungsmethode und anhand eines gemeinsamen Berichts des Ausschusses für Sozialschutz und des Ausschusses für Wirtschaftspolitik ... für die Frühjahrstagung 2002 des europäischen Rates gemeinsam einen exsten Bericht über Leitlinien im Bereich des Gesundheitswesens und Altenpllege (zu) erstellen. Die Ergebnisse der Beratung werden in den Grundzügen der Wirtschaftspolitik berücksichtigt."

Dieser Mitteilung der Kommission zur Zukunft der Gesundheitspolitik dürfte im Prozess des Agenda-settings im neuen europäischen Politikfeld Gesundheit aus mehreren Gründen wegweisende Bedeutung zukommen.

Im strategischen Ansatz knüpft sie an die Ausrichtung der Reformdebatten in den Mitgliedsstaaten an, in dem sie die gesundheitspolitische Problemanalyse auf die Sachkomplexe konzentriert, von denen nach allgemeiner Auffassung zukünftig erhebliche Kostensteigerungen ausgehen werden. Zum ersten Mal in dieser Ausführlichkeit beschreibt sie gemeinsame Problemlagen in den Gesundheitssystemen der Mitgliedsstaaten und leitet daraus eine Definition EUweiter versorgungspolitischer Ziele in der Gesundheitspolitik ab. Als Probleme benannt werden: die Überalterung der Bevölkerung, die Folgen der Entwicklung neuer Technologien und Therapien in der Medizin sowie die steigende Nachfrage der Bevölkerung nach medizinischer Versorgung und entsprechenden Angeboten im Zuge der Steigerung des Lebensstandards und des Bildungsniveaus. Zwar wird explizit die Zuständigkeit der Mitgliedsstaaten für die Organisation, für die Finanzierung der Gesundheitssysteme und für die auf die Bedürfnisse der Bevölkerung zugeschnittene Planung des Versorgungsangebotes festgestellt, um dann jedoch hinzu zu fügen:

„Diese Zuständigkeit wird aber innerhalb eines allgemeinen Rahmens ausgeübt, in den viele Gemeinschaftspolitiken immer stärker eingreifen. Aus diesem Grund ist die Entwicklung einer europäischen Zusammenarbeit auf diesem Gebiet erforderlich."

Mit Blick auf die Ziele dieser Zusammenarbeit werden drei gemeinsame Herausforderungen der Gesundheitssysteme in der europäischen Union (und den Kandidatenländern) benannt, die es gleichzeitig zu verwirklichen gelte. Zu diesen Zielen gehören zum einen die Sicherung des allgemeinen Zugangs zu einer hochwertigen Gesundheitsversorgung unter besonderer Berücksichtigung der Situation älterer Menschen, die der Langzeitpflege bedürfen; zum zweiten die Erhöhung der Transparenz und Qualität der Gesundheitssysteme insbesondere durch die Evaluierung von medizinischen Verfahren und Erzeugnissen sowie der Versorgungsstrukturen des Gesundheitswesens; und schließlich die Fortsetzung der auf Kostendämpfung abzielenden Reformen in Verbindung mit politischen Maßnahmen der Konsolidierung der öffentlichen Finanzen und zur Sicherung einer adäquaten Finanzierung der Gesundheitsvorsorge.

Insbesondere die Hervorhebung des Politikziels der Sicherstellung der langfristigen Finanzierbarkeit der Gesundheitssysteme lässt den wirtschafts- und fis- 
kalpolitischen Kontext deutlich werden, in den die europäische Gesundheitspolitik hinein formuliert wird. Dabei handelt es sich zweifelsfrei um die Strategie der rigiden Haushaltskonsolidierung im Rahmen des europäischen Stabilitäts- und Wachstumspaktes. Da sowohl dic staatlich organisierten Gesundheitssysteme als auch die parafiskalischen, öffentlich-rechtlichen Sozialversicherungen in die Berechnung der jährlichen Defizitquoten der öffentlichen Haushalte einbezogen werden, kann es kaum verwundern, dass dic finanzielle Entwicklung der Gesundheitssysteme insbesondere mit Blick auf eine potentielle Gefährdung des fiskalpolitischen Stabilitätsziels betrachtet wird. Betont wird, dass „gerade der Gesundheitspolitik eine entscheidende Bedeutung bei der Durchführung von Strategien zu (kommt), die nach Maßgabe der in den Grundzügen der Wirtschaftspolitik im Jahr 2001 aufgestellten Grundsätze (,Umschichtung der Staatausgaben zugunsten des Abbaus von Sach- und Humankapital' sowie ,effizienterer Einsatz der öffentlichen Mittel durch institutionelle und strukturelle Reformen') auf Qualität und Nachhaltigkeit setzen." Somit werden die wirtschaftspolitischen Prämissen einer auf öffentliche Haushaltskonsolidierung zielenden Reformstrategie sowie der Umschichtung von öffentlichen Ausgaben zugunsten des Aufbaus privatwirtschaftlicher Sachund Humankapitalstöcke auch als Leitlinien der Gesundheitspolitik implementiert.

Schließlich, und dies könnte für den vermuteten Aufbau einer europäischen Gesundheitspolitik von strategischer Bedeutung sein, insistiert die Mitteilung mit außerordentlicher Deutlichkeit auf den Beitrag der Gemeinschaftspolitiken für die europäische Gesundheitspolitik. Unter Berufung auf die Rechtssprechung des EuGH werden in einer eigenständigen Anlage ausführlich jene Regelungsgegenstände und Rechtsfelder des EG-Vertrages hervorgehoben, aus denen sich nach Auffassung der EU-Kommission implizite oder explizite $\mathrm{Zu}$ ständigkeiten der Gemeinschaft für den Bereich der Sozialpolitik ableiten lassen. So erinnert die EU-Kommission daran, dass es Aufgabe der Gemeinschaft sei, durch die Einrichtung eines gemeinsamen Marktes und einer Wirtschaftund Währungsunion sowie durch die Durchführung der gemeinsamen Politiken in der Gemeinschaft "ein hohes Beschäftigungsniveau und ein hohes Maß an sozialem Schutz ... zu fördern" (Art.2 EG-Vertrag). Dem entsprechend habe die Kommission in ihrer Mitteilung „Eine konzertierte Strategie zur Modernisierung des Sozialschutzes" (KOM 99347 endg.) „die Sicherung einer hohen Qualitätsansprüchen genügenden und langfristig finanzierbaren Gesundheitsversorgung zu einem der vier Hauptziele der europäischen Zusammenarbeit auf dem Gebiet der sozialen Sicherheit erklärt." Schließlich wird unmissverständlich auf die Garantien im Zuge der Freizügigkeit innerhalb des Waren-, Personen- und Dienstleistungsverkehrs verwiesen und hervorgehoben, dass nach Artikel 85 und 86 EG-Vertrag die Krankenkassen und die mit der Verwaltung von Systemen der sozialen Sicherheit betrauten Einrichtungen nur 
dann eine Aufgabe mir ausschließlich sozialem Charakter erfüllen, wenn ihre Tätigkeit auf dem Grundsatz der nationalen Solidarität beruht und nicht auf Gewinnerzielung ausgerichtet ist und wenn die zu erbringenden Leistungen gesetzlich festgesetzt und von der Höhe der entrichteten Beiträge unabhängig sind. Weichen Tätigkeiten im Bereich der Gesundheitsvorsorge von diesen Prinzipien ab und ließen sie sich als Wirtschaftstätigkeiten im Sinne des Vertrages qualifizieren, so der implizierte Hinweis, entstünden Problemkonstellationen des europäischen Wirtschaftsrechts, für die die Gemeinschaft allemal Zuständigkeit reklamieren könne.

In der deutschen Gesundheitspolitik könnte diesem „Wink mit dem Zaunpfahl ${ }^{\text {" }}$ besondere Bedeutung zukommen (Busse 2002). Insbesondere die politischen Akteure, die seit jeher auf Vermarktlichungs- und Deregulierungsstrategien im Gesundheitssystem setzten, könnten erheblichen politischen Aufwind erhalten (kritisch dazu: Deppe 2000, Deppe/Burghardt 2002). Damit dürften nicht nur die bereits wahrnehmbaren Prozesse einer wettbewerbskorporatistischen Restrukturierung des Gesundheitssystems an Bedeutung gewinnen (Urban 2001). Sollte sich über die neue Koordinierungsmethode das europäische Markt- und Wettbewerbsrecht stärkere Geltung verschaffen, könnte dies sehr schnell zu einer generellen Infragestellung des korporatistischen Regulierungsmodus und einer umfassenden Deregulierung des gesamten Vertrags- und Leistungssystems führen (Hirsch 2000, Hänlein/Kruse 2000). Wenn die Krankenkassen im Sinne des europäischen Wettbewerbsrecht als Wirtschaftsunternehmen aufgefasst würden, wären alle Verträge mit den Krankenhausgesellschaften, den Verbänden der Ärzte und den anderen Leistungsanbietern als wettbewerbswidrige Absprachen einzuschätzen. Sie würden ihre Wirksamkeit verlieren, und alles müsste über Marktbeziehungen gesteuert werde. Kommt dann noch der Primat von Kostensenkung und Privatisierung aus dem Euro-Finanzregime hinzu, und würde so der Druck auf das deutsche System verstärkt, wären die Tage des Solidarsystems gezählt.

\section{Der Übergang zu einem Neuen Europäischen Sozialmodell}

Auf diversen Nachfolgegipfeln ist der Anwendungsbereich der OMK auf nahezu alle wettbewerbspolitisch relevanten Politikfelder ausgeweitet worden. Über das vereinbarte Benchmarking- und Überwachungsverfahren könnte sich eine erhebliche Dynamik entfalten, die nicht nur eine reale Einschränkung der Autonomie der Nationalstaaten bedeutet, sondern auch die nationalen Wohlfahrtsreformen in Richtung eines wettbewerbsorientierten Umbaus der nationalen Systeme strukturiert. Denn das gesamte Verfahren der offenen Koordinierung bleibt eng in die wettbewerbs- und stabilitätspolitischen Vorgaben des Euro-Finanzregimes eingebunden. Das strategische Kernziel besteht darin, die Felder der Sozialpolitik nach seinen Kriterien auszurichten. Und das bedeutet 
in erster Linie: Kostensenkungen, Verschärfung von Anspruchsvoraussetzungen, Flexibilisierung und - nicht zuletzt - die Öffnung der Felder des sozialen Schutzes für die Einkommens- und Verwertungsinteressen der Finanzmarktakteure. Es spricht viel dafür, dass diese Einbindung des gesamten Verfahrens politische Reformoptionen der Mitgliedstaaten, die nicht reibungslos kompatibel mit diesem Kontext sind, erheblich erschwert und andere weiter befördert werden.

Ein solcher Umbau der sozialen Sicherung findet nicht nur in Deutschland, sondern in vielen Staaten der EU statt. Und immer stärker gewinnen Strategien, die den Finanzmarktakteuren einen Zugang in diese sozialstaatlichen Politikfelder organisieren, an Bedeutung. Allmählich werden auf nationalstaatlicher wie europäischer Ebene die Konturen eines Neuen Europäischen Sozialmodells sichtbar (Aust/Leitner/Lessenich 2002), das sich geradlinig in die wettbewerbspolitische Formierung des Wirtschaftsraums Europa im Sinne der Lissabonner Strategie einpasst. In diesem Modell gibt es auch weiterhin Sozialpolitik, aber eine, die zur Sicherung der sozialen Bürgerrechte und zum Schutz gegenüber den Zumutungen der kapitalistischen Marktwirtschaft nicht mehr viel beitragen dürfte. M.E. wird sich das Neue Europäische Sozialmodell von der bisherigen Variante des sozialstaatlich regulierten Kapitalismus in Europa zumindest in folgenden Elementen unterscheiden:

- Der Sozialpolitik der Nationalstaaten und der EU wird nicht mehr die Rolle zugewiesen, die im Kapitalismus chronisch ungleiche Verteilung von Einkommen, Vermögen und Lebenschancen zu korrigieren sowie Wettbewerb und Markt zu regulieren. Vielmehr mutiert Sozialpolitik allmählich zu einer Variante von Wettbewerbspolitik. Die sozialpolitischen Programme und Ziele der EU zielen vor allem auf die Verbesserung der Angebotsbedingungen der Unternehmen, etwa über die Anhebung der Qualifikation und des Gesundheitszustandes der Beschäftigten oder die schnellere Vermittlung von Arbeitslosen. Es geht vor allem um Beiträge zur betrieblichen Wettbewerbsfähigkeit und zur volkswirtschaftlichen Wertschöpfung.

- Zugleich geht die Herausbildung des Neuen Europäischen Sozialmodells auch mit einer Neubewertung der "sozialen Frage" einher. Galten bisher soziale (Verteilungs-) Gerechtigkeit und Gleichheit in den Lebenschancen als Ziele des Europäischen Sozialmodells, so hat die Neue Sozialdemokratie in Europa auf ihrem „Dritten Weg“ die angeblich wettbewerbs- und produktivitätsfördernde Funktion sozialer Ungleichheit entdeckt (Mahnkopf 2000). Soziale Gerechtigkeit und Gleichheit werden als potentiell totalitär und standortschädlich diffamiert und die Re-Polarisierung der Verteilung von Einkommen, Vermögen und Lebenschancen wird zum dezidierten Politikziel.

- Der Dominanz von Markt und Wettbewerb hat sich auch die Vorstellung von Solidarität unterzuordnen. Solidarität heißt nicht länger, die Konkurrenz unter den Lohnabhängigen außer Kraft zu setzen; und auch nicht, Unternehmen 
und hohe Einkommensgruppen über Steuern oder Sozialversicherungsbeiträge zu belasten, um daraus Systeme des sozialen Schutzes zu finanzieren. Der neue Begriff von Solidarität empfiehlt den sozialen Konfliktparteien, sich auf betrieblicher wie auf gesellschaftlicher Ebene zu Wettbewerbskoalitionen zusammenschließen, um im inländischen oder im globalen Konkurrenzkampf besser überleben zu können. Nicht mehr die soziale Klasse, sondern der Betrieb oder der Wirtschaftsstandort sollen die neuen Solidar-Gemeinschaften bilden (Streeck 2000).

- Diese „Wettbewerbssolidarität" ("Competitive Solidarity“, Wolfgang Streeck) dient zugleich zur Begründung und Rechtfertigung der empfohlenen oder geforderten "institutionalisierten Wettbewerbskoalitionen " bzw. „Sozialpakte" (Hassel 2000, Traxler 2001). Diese werden in den Betrieben zwischen der dortigen Interessenvertretung und der Unternehmensleitung, in der Gesellschaft zwischen den Verbänden von Kapital und Arbeit sowie dem Staat geschlossen. Im Kern geht es um die Einbindung der Gewerkschaften in eine wettbewerbskorporatistische Modernisierungsstrategie, die letztlich den Übergang zum finanz(markt)getriebenen Entwicklungsmodell zu organisieren versucht. Für die Gewerkschaften eine höchst prekäre Angelegenheit, die sie schnell in die politische Subalternität abzudrängen oder in heftige Konflikte zwischen Vorständen und Mitgliedschaft zu führen droht (Urban 2001, Bieling/Schulten 2002).

- Doch auf eine Gegenleistung in Form einer alktiven Wirtschaftspolitik gegen Wachstumskrise und Arbeitslosigkeit brauchen die Gewerkschaften nicht zu hoffen. Im Neuen Sozialmodell herrschen nicht nur die Paradigmen angebotspolitischer und monetaristischer Wirtschaftstheorien (Huffschmid 1999). Auch die Finanz- und Geldpolitik wird durch die Vorgaben des Stabilitätspaktes sowie die Aufgabenbestimmung der europäischen Zentralbank weitgehend entwertet (Oberhauser 1996, Priewe 1997). Damit muss dieses Modell ohne eine aktive Konjunktur-, Wachstums- und Beschäftigungspolitik auskommen. In der europäischen Wirtschaftspolitik stellt der Policy-Mix des „Makroökonomischen Dialoges" zwar gegenüber einer wechselseitigen Anpassungskonkurrenz einen Fortschritt dar. Doch letztlich versucht er eben auch, die Gewerkschaften auf eine „zurückhaltende“ Tarifpolitik zu verpflichten, um angeblichen lohninduzierten Inflationsgefahren und entsprechenden Zinsreaktionen der EZB vorzubeugen.

Mit diesen Elementen sind sicher nicht alle Stützen des Neuen Europäischen Sozialmodells beschrieben. Sie genügen aber, um deutlich werden zu lassen: Sollte sich dieses Leitbild eines Neuen Sozialmodells in die Realität umsetzen, hätten Lohnabhängige, Gewerkschaften und die politische Linke in Europa nichts Gutes davon zu erwarten. Die eindeutige Unterordnung von Sozialpolitik und gesellschaftlichen Gerechtigkeitszielen unter die Zwänge einer aggressiven Wettbewerbspolitik bzw. ihre strukturelle Abwertung im Übergang zu einem neuen sozialökonomischen Entwicklungsmodell dürften kaum einen Bei- 
trag zur Beseitigung von struktureller Massenarbeitslosigkeit, zunehmender Polarisierung in der Verteilung des gesellschaftlichen Reichtums und der voranschreitenden Marginalisierung gesellschaftlicher Minderheiten leisten.

\section{Anforderungen an ein strategisches Gegenprojekt der europäischen Linken}

Es lassen sich wohl kaum überzeugende Argumente gegen die These in Anschlag bringen, dass die Linke in Europa eine klare Oppositionshaltung gegenüber diesem neuen „Sozialmodell“ einnehmen sollte. Wie sich die sozialistischen bzw. sozialdemokratischen Parteien in Europa gegenüber den strategischen Prämissen dieses Projektes positionieren, scheint noch nicht endgültig ausgemacht. Einerseits dominiert in vielen dieser Parteien die Philosophie des „Dritten Weges", der geradeaus in das Neue Europäische Sozialmodell hinein führt. Und dies ist kein Zufall: Das Neue Sozialmodell ist das Modell der Neuen Sozialdemokratie. Doch andererseits scheinen allmählich auch die internen politischen Oppositionskräfte an Selbstbewusstsein zu gewinnen, die auf eine andere, fortschrittliche Modernisierung Europas drängen.

Einen ähnlichen Streit um die zukünftige strategische Ausrichtung gibt es in den europäischen Gewerkschaften. So können die gewerkschaftlichen Proteste der jüngsten Vergangenheit in Deutschland, Frankreich, Österreich, Italien usw., die sich insbesondere gegen den Umbau der sozialen Sicherungssysteme nach den Prämissen der Lissabonner Wettbewerbsstrategie wandten, durchaus als Rebellionen gegen die nationalstaatlichen Umsetzungsinitiativen des neuen europäischen Sozialmodells gelten. Unterstützend könnten sich für die Linke in den Gewerkschaften und Parteien das langsame, aber doch sichtbar werdende Wiederaufleben sozialer Protestbewegungen und Selbsthilfeinitiativen sowie die beeindruckende transnationale Vernetzung der Bewegung der Globalisierungskritiker erweisen. Welchen politischen Handlungsfeldern und strategischen Reformprojekten welche Bedeutung in einem linken Gegenprojekt zukommen könnte, bliebe einem anstehenden, pluralistischen Diskussionsund Verständigungsprozess vorbehalten. Dazu einige Überlegungen.

Ein linkes Benchmarking-Verfahren? Soll es gelingen, dem gegenwärtigen gesundheitspolitischen Integrationsprozess eine andere Richtung zu gebe, muss die Linke eine eigene Vorstellung davon entwickeln, auf welchen Entwicklungspfad sich Europa begeben soll. Das gilt nicht zuletzt für die gesundheitliche Versorgung. Hier wäre zu fragen, ob der „Benchmarking"-Gedanke der offenen Koordinierungsmethode, trotz aller Defizite (dazu: Urban 2003), nicht auch für solidarische Reformprojekte nutzbar wäre. Ein „linkes Benchmarking-Verfahren“" würde allerdings nicht nach „bewährten Praktiken" der Kostensenkung, Privatisierung und Kapitalisierung der sozialen Sicherungssysteme Ausschau halten. Es müsste darauf orientieren, wo in europäischen Län- 
dern gelungene Beispiele für eine solidarische Reform der sozialen Sicherheitssysteme zu finden sind. Und es müsste danach fragen, wie diese guten Beispiele in den nationalstaatlichen Auseinandersetzungen politik- und durchsetzungsfähig gemacht werden könnten.

Transformation des Euro-Finanzregimes. Welche Spielräume für eine solidarische Modernisierung der europäischen Wohlfahrtsstaaten und des Europäischen Sozialmodells zur Verfügung stehen, wird nicht zuletzt durch die Ausgestaltung des Euro-Finanzregimes entschieden. Sollte es bei der jetzigen Form des Stabilitätspaktes und der Rolle der Europäischen Zentralbank bleiben, ist eine Wende in Richtung auf mehr Wachstum, Beschäftigung und soziale Gerechtigkeit in Europa nicht zu erwarten. Die Defizitkriterien des Stabilitätspaktes müssen grundlegend verändert werden; und die europäische Zentralbank muss mit ihrer Geldpolitik zusätzlich auf das Ziel der Förderung von wirtschaftlichem Wachstum und Beschäftigung verpflichtet werden. In der jüngeren Vergangenheit häufen sich die Anzeichen dafür, dass Bewregung in die Debatte über den Stabilitätspakt kommt. Dies vor allem, weil sich die Vorgaben des Euro-Regimes immer offensichtlicher als Blockaden für die Überwindung der aktuellen Wachstumskrisen, Staatsdefizite und Beschäftigungsprobleme erweisen. Die Linke muss sich hier einschalten und ihre Vorstellungen für eine grundsätzliche Veränderung des Euro-Finanzregimes offensiv zur Geltung bringen (dazu etwa: Europäische Memorandum-Gruppe 2003).

Demokratisierung des offenen Koordinierungsverfahrens. Darüber hinaus wäre zu fragen, welche Akteure zu welchem Zeitpunkt und mit welchen politischen Inhalten in die Abwicklung des Verfahrens der offenen Koordinierung intervenieren könnten und sollten. Das formale Beteiligungsangebot ist bisher weitgehend Theorie geblieben. Weder die Gewerkschaften noch andere Akteure der Bürgergesellschaft sind an den aktuellen Prozessen mit Einfluss beteiligt. Auch am europäischen sowie an den nationalen Parlamenten läuft das ganze Verfahren weitgehend vorbei. Demgegenüber scheinen die Institutionen der europäischen Exekutive, die Europäische Kommission und der Europäische Rat, ihre Machtposition in diesem Verfahren auszubauen. Dies geschieht weitgehend ohne Proteste und Widerstand. Insgesamt scheinen die potenziellen Akteure einer Gegenreform, die nationalen Parlamente, die Gewerkschaften, die sozialen Initiativen und Protestbewegungen ihre Rolle in diesem neuen Koordinierungsverfahren noch nicht gefunden zu haben. In Deutschland beginnen einige, wenige Akteure erst ganz langsam zu begreifen, was da über das strategische Leitbild der Lissabonner Wettbewerbsstrategie und das Vehikel der OMK mit der Sozialpolitik in Europa geschieht und was auf die nationalen Wohlfahrtsstaaten zukommt. Eine öffentlichkeitswirksame Debatte wäre dringend notwendig. 
Chancen einer solidarischen Gesundheitspolitik. Bliebe die Frage, wie den Implikationen der Europäisierung der Gesundheitspolitik über die OMK im Prozess der Reformierung des deutschen Gesundheitssystems adäquat zu begegnen wäre. An dieser Stelle kann den einzelnen Wirkungskanälen, über die sich der wettbewerbspolitische Umbau des europäischen Sozialmodells im deutschen Gesundheitssystem Geltung verschafft, nicht weiter nachgespürt werden (ausführlich dazu: Urban 2003). Es kann aber kaum ein Zweifel daran bestehen, dass die enge Einbindung der europäischen Gesundheitspolitik in die finanzpolitischen Vorgaben des Euro-Finanzregimes und die wettbewerbspolitischen Ziele der Lissabonner Strategie Druck in Richtung der Stärkung einer kostendämpfungs- und wettbewerbsorientierten Politik entfalten wird. Dies spielt jenen Alkteuren im deutschen Gesundheitssystem in die Hände, die auch ohne diese Unterstützung auf Strategien der Kürzung des Leistungskataloges der Krankenversicherung, Privatisierung ehemals unter Beteiligung der Arbeitgeber finanzierter Leistungen sowie die Implementierung weiterer Marktund Wettbewerbselemente in das gesundheitspolitische Steuerungssystem setzen. Doch sollten sich im deutschen Gesundheitssystem die Versuche verstetigen, der europäisch verstärkten Kostensenkungs- und Vermarktlichungsperspektive durch die Ausdehnung wettbewerblicher und risikoprivatisierender Regelungen zu genügen (kritisch dazu: Gerlinger/Lehnhard/Stegmüller 2002), so würde dies zwar einem „internationalen Megatrend" in der Gesundheitspolitik (Buchner/Wasem 2000: 361) folgen. Doch zugleich wären gravierende Folgeprobleme abzusehen. Denn im Gesundheitsversorgungssystem eignen sich Markt und Wettbewerb weder als Instrumente zur Kostendämpfung noch zur Verbesserung der Versorgungsqualität; beide neigen vielmehr eher dazu, zu paradoxen Politikergebnissen zu führen, die vielfach mit den anvisierten Politikergebnissen nicht mehr viel zu tun haben oder gar in ihr Gegenteil umschlagen. So lassen internationale Studien kaum mehr Zweifel daran, dass durch die Implementierung wettbewerblicher Steuerungselemente Umfang und Kosten der medizinischen Versorgung nicht sinken sondern eher expandieren, dass durch die Privatisierung der Versorgung die soziale Ungleichverteilung von Krankheitsrisiken und Gesundheitschancen eher größer als kleiner wird und dass sich insgesamt ohne staatliche Interventionen in den Prozess der Produktion, Verteilung und Konsumtion von Gesundheitsleistungen und gütern mehr Effizienz, Effektivität und Qualität nicht realisieren lassen (dazu ausführlicher Urban 2003).

Welche Optionen mit Blick auf die Zukunftssicherung eines leistungsfähigen, sozialen und solidarischen Gesundheitssystems die richtigen sein könnten, bedarf sicherlich einer intensiven Debatte (zur aktuellen Reformdebatte vgl. Deppe/Burghardt 2002). Hier mag der Hinweis genügen, dass die Problemkonstellation, die durch die Kollision zwischen europäischem Wettbewerbs- und deutschem Sozialrecht hervorgerufen wird, sich auch aus einer Perspektive in- 
terpretieren lässt, die die Solidarelemente im deutschen System nicht schwächt, sondern stärken könnte. Die Herausnahme der sozialen Krankenversicherung aus dem europäischen Marktrecht beruht - wie dargelegt - darauf, dass sie Aufgaben mit ausschließlich sozialem Charakter erfüllt. Dies ist aber nur dann und solange der Fall, wie sie auf dem Grundsatz der Solidarität beruht und nicht auf Gewinnerzielung ausgerichtet ist, sie also sowohl durch ihre Finanzierung als auch durch ihre Leistungsgewährung sozial umverteilend wirkt, und solange sie gesetzlich festgelegte und von der individuellen Beitragshöhe unabhängige Leistungen erbringt. Nur in diesem Fall stellt ihre Tätigkeit keine Wirtschaftstätigkeit dar und sind die Träger der sozialen Krankenversicherung nicht als Unternehmen anzusehen. Dies könnte eben auch als Orientierungshilfe für eine adäquate Reformstrategie interpretiert werden. Denn durch diese Konstellation werden Reformen im Sinne von mehr Wettbewerb und Privatisierung enge Grenzen gesetzt. Dies insbesondere in Bezug auf die im politischen Sektor vielfach präferierten Optionen der Zurückdrängung von internen Solidarelementen zugunsten versicherungsförmiger Äquivalenzelemente, der Ausdifferenzierung des gesetzlichen Leistungskataloges in paritätisch finanzierte Basis- und private Zusatzleistungen oder der Einführung von Regelungen aus der privaten Krankenversicherung (Bieback 1999, Ebsen 2000).

Mit anderen Worten: die Europäisierung der Gesundheitspolitik muss keineswegs ausschließlich als Bedrohung für den Solidarcharakter des deutschen Gesundheitssystems wahrgenommen werden. Aus ihr lassen sich gleichfalls zusätzliche Begründungen für seinen Erhalt ableiten. Wer also nicht das ganze Steuerungsmodell mit seinen Aushandlungs- und Vertragsbeziehungen grundsätzlich zur Disposition stellen will, muss äußerst bedacht mit der Forderung nach mehr Markt und Wettbewerb im Gesundheitssystem umgehen. Einmal mehr wird deutlich, dass das Hantieren mit Wettbewerbs- und Privatisierungselementen ordnungspolitisch einem Spiel mit dem Feuer gleichkommt. Im Falle einer Unterwerfung der sozialen Krankenversicherung unter die Ägide des europäischen Wettbewerbsrechts und das Regime des freien Waren- und Dienstleistungsverkehrs wäre es binnen kurzer Zeit um den sozialstaatlichen Charakter des Gesundheitssystems geschehen und wäre weiteren Ökonomisierungsschüben Tür und Tor geöffnet.

\section{Literatur}

Albert, Michel (2001): Kapitalismus contra Kapitalismus. Zehn Jahre danach, in: Blätter für deutsche und internationale Politik H. 12, S. 1451-1462.

Aglietta, Michel (2000): Ein neues Akkumulationsregime. Die Regulationstheorie auf dem Prïfstand. Hamburg.

Aust, Andreas/Leitner, Sigrid/Lessenich, Stephan (2002): Konjunkîur und Krise des Europäischen Sozialmodells, in: Politische Vierteljahresschrift H. 2, S. 272-301.

Bieback, Karl-Jurgen (1999): Rechtliche und politische Dimensionen der EU-Gesundheitspolitik und des Zugangs der Bürger zu Gesundheitsleistungen, in: Zeitschrift fïr Sozialreform, H. 8, S. 663-692. 
Bieback, Karl-Jürgen (2001): Die Stellung der Sozialleistungserbringer im Marktrecht der EG, in: Beiträge zum Recht der sozialen Dienste und Einrichtungen. Bd. 49, S. 1-39.

Bieling, Hans-Jürgen/Steinhilber, Jochen (Hrsg.) (2000a): Die Konfiguration Europas. Dimensionen einer kritischen Integrationstheorie. Münster.

Bieling, Hans-Jürgen/Steinhilber, Jochen (2000b): Hegemoniale Projekte im Prozess der europäischen Integration, in: Dies. (2000a), S. 102-130.

Bieling, Hans-Jürgen u.a. (Hrsg.) (2001): Flexibler Kapitalismus. Hamburg.

Bieling, Hans-Jürgen/Steinhilber, Jochen (2002): Finanzmarktintegration und Corporate Governance in der Europäischen Union, in: Zeitschrift für internationale Beziehungen, H.1, S. 39-74.

Bieling, Hans-Jürgen/Schulten, Thorsten (2002): Reorganisation der industriellen Beziehungen im europäischen Mehrebenensystem, in: Industrielle Beziehungen, H. 3, S. 245-273.

Boyer, Robert (2000): Is a finance-led growth regime a viable alternative to Fordism? A preliminary analysis, in: Economy and Society, H.1, S. 111-145.

Buchner, Florian/Wasem, Jürgen (2000): Wettbewerb zwischen Krankenversicherungsträgern als Steuerungsinstrument der Gesundheitsversorgung. Eine internationale Perspeltive, in: Zeitschrift für Sozialreforn, H. 4, S. 353-363.

Busse, Reinhard (2002): Anwendung der „offenen Methode der Koordinierung“ auf die europäischen Gesundheitswesen. Hintergründe, mögliche Ziele und Indikatoren, Auswirkungen auf Gesundheitssysteme, in: $G^{+} G$-Wissenschaft H.2, S. 7.4.

Deppe, Hans-Ulrich (2000): Die soziale Anatomie des Gesundheitswesens. Neoliberalismus und Gesundheitspolitik in Deutschland. Frankfurt/M.

Deppe, Hans-Ulrich / Burghardt, Wolfram (Hrsg.) (2002): Solidarische Gesundheitspolitik. Alternativen zu Privatisierung und Zwei-Klassen-Medizin. Hamburg.

Dudey, Stefan/ Hesse, Sebastian/Jacobs, Klaus/Wasem, Jürgen (2002): Weiterentwicklung einer leistungsfahigen und solidarischen Krankenversicherung unter den Rahmenbedingungen der europäischen Integration. Endbericht für ein von der Hans-Böckler-Stiftung gefördertes Forschungsvorhaben. November (unveröffentlichtes Manuskript).

Ebsen, Ingwer (2000): Öffentlich-rechtliches Handeln von Krankenkassen als Gegenstand des Wettbewerbsrechts? Probleme materialrechtlicher und kompetenzrechtlicher Koordinierung, in: Zeitschrift für Sozialreform, H. 4, S. 298-314.

Eckardt, Martina (2002): Die Methode der offenen Koordinierung. Konsequenzen für die Alterssicherung(spolitik) in der Europäischen Union, in: Sozialer Fortschritt, H. 7-8, S. 197-203.

Europäische Memorandum-Gruppe (Hrsg.)(2003): EuroMemo. Bessere Institutionen, Regeln und Instrumente für Vollbeschäftigung und sozialen Wohlstand in Europa. Hamburg.

Europäischer Rat (2000): Schlussfolgerungen des Vorsitzes. Europäischer Rat vom 23. und 24. März 2000, Lissabon.

Falkner, Gerda (1998): EU Social Policy in the 1990s: Towards a corporatist policy community. London, New York.

Hänlein, Andreas/Kruse, Jürgen (2000): Einflüsse des Europäischen Wettbewerbsrechts auf die Leistungen der gesetzlichen Krankenversicherung, in; Neue Zeitschrift fir Sozialrecht, H. 4, S. 165-176.

Hassel, Anke (2000): Bündnisse für Arbeit: nationale Handlungsfähigkeit im europäischen Regimewettbewerb, in: Politische Vierteljahresschrift, H. 3, S. 498-524.

Hill, Hermann (2002): Zur ,Methode der offenen Koordinierung“ in der Europäischen Union, in: Sommermann, Karl-Peter (Hrsg.): Perspektiven der Verwaltungsforschung: Beiträge zur wissenschaftlichen Arbeitstagung aus Anlass des 25-jährigen Bestehens des Forschungsinstituts für offentliche Verwaltung vom 8. bis 10. Oktober in Speyer. Berlin, S. 139-162.

Hirsch, Günter (2000): Einfluß der EG auf nationale Gesundheitssysteme, in: Medizinrecht, H. 12, S. 586-590.

Hodson, Dermont/Maher, Imelda (2001): The Open Method as a New Mode of Governance: The Case of Soft Economic Policy Co-ordination, in: Journal of Common Market Studies, No. 4, S. 719-746.

Huffschmid, Jörg (1999): Politische Ökonomie der Finanzinärkte. Hamburg.

Jachtenfuchs, Markus/ Kohler-Koch, Beate (Hrsg.) (1996): Europäische Integration. Opladen.

Kommission der europäischen Gemeinschaft (EU-Kommission) (2001): Die Zukunft des Gesundheitswesens und der Altenpflege: Zugänglichkeit, Qualität und langfristige Finanzierbarkeit sichern. Mitteilung der Kommission an den Rat, das Europäische Parlament, den Wirtschaftsund Sozialausschuss und den Ausschuss der Regionen, Brüssel, KOM (2001) 723 endg.. 
Kraus, Katrin/Geisen, Thomas (Hrsg.): Sozialstaat in Europa. Geschichte, Entwicklung, Perspektiven. Opladen 2001.

Leibfried, Stephan (2000): Nationaler Wohlfahrtsstaat, Europäische Union und "Globalisierung". Erste Annäherungen, in: Allmendinger, Jutta/Ludwig-Mayerhofer, Wolfgang (Hrsg.): Soziologie des Sozialstaats. Gesellschaftliche Grundlagen, historische Zusammenhänge und aktuelle Entwicklungstendenzen. Weinheim, München 2000, S. 79-108

Leibfried, Stephan/Pierson, Paul (Hrsg.)(1998): Standort Europa. Sozialpolitik zwischen Nationalstaat und Europäischer Integration. Frankfurt/M.

Mahnkopf, Birgit (2000): Formel 1 der neuen Sozialdemokratie: Gerechtigkeit durch Ungleichheit. Zur Neuinterpretation der sozialen Frage im globalen Kapitalismus, in: PROKLA 121, S. 489-525.

Oberhauser, Alois (1996): Geknebelte Beschäftigungspolitik - Eine Folge der Maastricht-Kriterien, in: Mitteilungen aus der Arbeitsmarkt- und Berufsforschung, H. 2, S. 228-236.

Ohndorf, Wolfgang (2002): Die „offene Koordinierung“ als Handlungsinstrument auf Europäischer Ebene. Einführung, in: Verband Deutsche Rentenversicherungsträger u.a. (Hrsg.): S. 16-20.

Pitschas, Rainer (2002): Nationale Gesundheirsreform und europäische "Governance" in der Gesundheitspolitik. Zur Verpflichtung der Gemeinschaftsstaaten auf Solidarischen Wettbewerb durch „offene Koordination“, in: Vierteljahresschrift für Sozialrecht, H. 2, S. 75-91

Priewe, Jan ( 1997): Verschuldungsregeln in der Europäischen Union, in. WSI Mitteilungen H. 6 , S.365-373.

Rosenbrock, Rolf/Gerlinger, Thomas (2003): Gesundheitspolitik. Eine systematische Einführung. Bem.

Sachverständigenrat zur Begutachtung der gesamtwirtschaftlichen Entwicklung (Sachverständigenrat 2002): Jahresgutachten 2002/2003. Wiesbaden.

Scharpf, Fritz W. (1985): Die Politikverflechtungs-Falle. Europäische Integration und deutscher Förderalismus im Vergleich, in: Politische Vierteljahresschrift, H. 4, S. 323-356.

Scharpf, Fritz W. (1994): Autonomieschonend und gemeinschaftsverträglich: Zur Logik einer europäischen Mehrebenen-Politik, in: Ders., Optionen des Föderalisinus in Deutschland und Europa. Frankfurt a. M., New York, S. 131-155.

Scharpf, Fritz W. (1999): Regieren in Europa. Effektiv und demokratisch? Frankfurt/M., New York.

Schulte, Bernd (2001): Europäische Integration und sozialer Schutz, in: Kraus/Geisen (Hrsg.): S. 284 303.

Schulte, Bernd (2002): Die „Methode der offenen Koordinierung“. Eine politische Strategie in der europäischen Sozialpolitik auch für den Bereich des sozialen Schutzes, in: Zeitschrift für Sozialreform, H. 1, S. 1-28.

Schulz-Weidner, Wolfgang (2002): Offene Koordinierung der sozialen Sicherungssysteme in der Europäischen Union. Statement auf dem VDR Pressekontaktseminar 2002 (unv. Manuskript).

Streeck, Wolfgang (1998): Vom Binnenmarkt zum Bundesstaat? Überlegungen zur politischen Ökonomie der europäischen Sozialpolitik, in: Leibfried/Pierson, S. 369-421.

Streeck, Wolfgang (2000): Competitive Solidarity: Rethinking the „European Social Model", in: Hinrichs, Karl/Kitschelt, Herbert/Wiesenthal, Helmut (Hrsg.): Kontingenz und Krise. Institutionenpolitik in der kapitalistischen und postsozialistischen Gesellschaft. Frankfurt/New York, S. 245-261.

Traxler, Franz (2001): Die Metamorphose des Korporatismus: Vom klassischen zum schlanken Muster, in: Politische Vierteljahresschrif, H. 4, S. 590-623.

Urban, Hans-Jürgen (Hrsg.)(2000): Beschäftigungsbündnis oder Standortpakt. Das „Bündnis für Arbeit" auf dem Prüfstand. Hamburg.

Urban, Hans-Jürgen (2001): Wettbewerbskorporatistische Regulierung im Politikfeld Gesundheit. Der Bundesausschuss der Arzte und Krankenkassen und die gesundheitspolitische Wende. Wissenschaftszentrum Berlin für Sozialforschung, Arbeitsgruppe Public Health, Discussion Paper, P. 01/206, Berlin.

Urban, Hans-Jürgen (2003): Europäisierung der Gesundheitspolitik. Zur Evolution eines Politikfeldes im europäischen Mehrebenen-System. Wissenschaftszentrum Berlin für Sozialforschung, Arbeitsgruppe Public Health, Discussion Paper. (i.E.).

Verband Deutscher Rentenversicherungsträger/Bundesministerium für Arbeit und Sozialordnung/MaxPlanck-Institut für internationales und ausländisches Sozialrecht (Hrsg.)(2002): Offene Koordinienung der Altersicherung in der Europäischen Union (DRV-Schriften, Bd. 34): Frankfurt/M. 